\title{
Automated Tessellated Fundus Detection in Color Fundus Images
}

\author{
Mengdi $\mathrm{Xu}^{1}$, Jun Cheng ${ }^{1}$, Damon Wing Kee Wong ${ }^{1}$, Ching-Yu Cheng ${ }^{2}$, Seang \\ Mei Saw ${ }^{2}$, and Tien Yin Wong ${ }^{2}$ \\ 1 Institute for Infocomm Research, Agency for Science, Technology and Research, \\ Singapore (E-mail: xumd@i2r.a-star.edu.sg) \\ 2 Singapore Eye Research Institute
}

\begin{abstract}
In this work, we propose an automated tessellated fundus detection method by utilizing texture features and color features. Color moments, Local Binary Patterns (LBP), and Histograms of Oriented Gradients (HOG) are extracted to represent the color fundus image. After feature extraction, a SVM classifier is trained to detect the tessellated fundus. Both linear and RBF kernels are applied and compared in this work. A dataset with 836 fundus images is built to evaluate the proposed method. For linear SVM, the mean accuracy of $98 \%$ is achieved, with sensitivity of 0.99 and specificity of 0.98 . For RBF kernel, the mean accuracy is $97 \%$, with sensitivity of 0.99 and specificity of 0.95 . The detection results indicate that color features and texture features are able to describe the tessellated fundus.
\end{abstract}

Keywords: Tessellated fundus, image classification, texture features

\section{INTRODUCTION}

High myopia with any posterior myopia-specific pathology from axial elongation is called pathologic myopia [1]. Pathologic myopia can causes a major alteration of the globe or shape of the eye. These changes in eyes with pathologic myopia are termed myopic maculopathy, which is one of the major causes of legal blindness worldwide [2].

Recently, Matsui et al. [2] conducted a study on pathologic myopia, which called META-PM (Meta-Analysis for Pathologic Myopia). In their work, five categories of myopic maculopathy are defined and studied, they are:

- No myopic retinal degenerative lesion (Category 0)

- Tessellated fundus (Category 1)

- Diffuse chorioretinal atrophy (Category 2)

- Patchy chorioretinal atrophy (Category 3)

- Macular atrophy (Category 4)

In this work, we focus on the detection of tessellated fundus. The tessellated fundus related to myopia can be defined to have choroidal vessels around the

X. Chen, M. K. Garvin, J. Liu, E. Trucco, Y. Xu (Eds.): OMIA 2016, Held in Conjunction with MICCAI 2016, Athens, Greece, Iowa Research Online, pp. 25-32, 2016. Available from: http://ir.uiowa.edu/omia/2016_Proceedings/2016/ 
arcade vessels and the fovea [2]. These choroidal vessels are well-defined and can be clearly observed. Fig. 1 shows some standard images with tessellated fundus.

Currently, the detection of tessellated fundus is conducted by ophthalmologist manually. Manual assessment is quite time-consuming, thus an automatic method would be necessary.

To the best of our knowledge, there is no existed work on fully automated tessellated fundus detection. But there are some works on automated detection of other signs of myopia. As one of the early signs of myopia, peripapillary atrophy (PPA) have been studied recently. Several automatic PPA detection approaches have been proposed. Tan et al. [3] proposed a disc difference approach. The difference between optic disc region and the region of optic disc inclusive of the PPA was computed. The presence of PPA was defined based on the asymmetry of the difference. Cheng et al. [4] presented an automatic PPA detection method utilizing biologically inspired feature (BIF). Sparse transfer learning was explored to reduce feature dimensionality. The proposed approach achieved an accuracy of more than $90 \%$ in PPA detection.
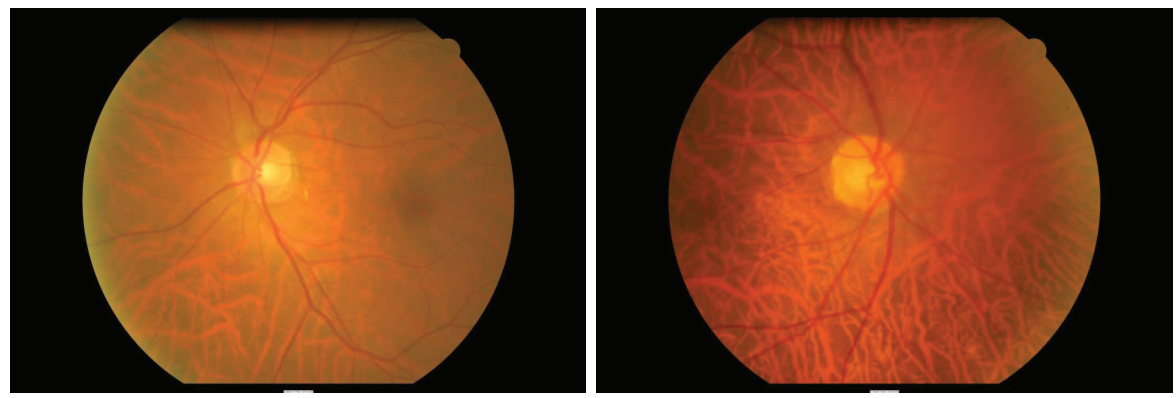

Fig. 1. Standard photographs with tessellated fundus.

In this work, we propose an automated tessellated fundus detection method by utilizing color moments, LBP and HOG features. Color moments measures the color features by computing the mean and standard deviation values on R, G, and B. Histograms of Oriented Gradients (HOG) [5] captures the edge information, and Local Binary Patterns (LBP) descriptor [6] describes texture. These two features are widely used in face detection, image classification, etc.

This paper is organized as follows. Section 2 introduces methodology and features we used in the experiment. Section 3 shows experimental results. Discussion and conclusions are introduced in Section 4.

\section{METHODOLOGY}

This section introduces technical details of the proposed automated tessellated fundus detection method. The proposed method includes following main steps: a) 

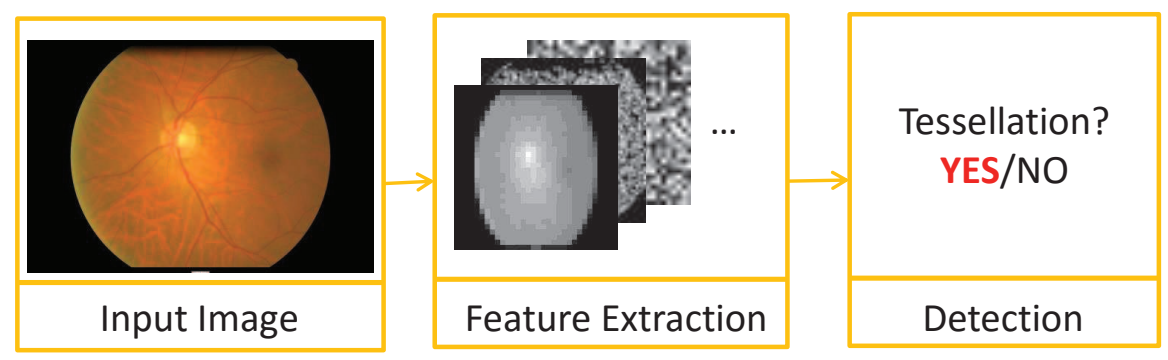

Fig. 2. Pipeline of the proposed method.

Feature extraction to get color and texture features, and b) Detection to detect the presence of tessellation. Fig. 2 shows the pipeline of the proposed method.

\subsection{Feature Extraction}

In this work, we extract color moments, Histograms of Oriented Gradients (HOG) [5] and Local Binary Patterns (LBP) [7] bag-of-words (BOW) [8] features to represent the color fundus images. Fig. 3 shows the process of feature extraction.

Color Moments Color moments features measure the feature of color. We compute the mean value and standard deviation of $\mathrm{R}, \mathrm{G}, \mathrm{B}$ of the color fundus image as the color moments. Thus it could be represented as a 6 -D vector, $C=[\operatorname{mean}(R), \operatorname{mean}(G), \text { mean }(B), \operatorname{std}(R), \operatorname{std}(G), \operatorname{std}(B)]^{\prime}$.

Local Binary Patterns The Local Binary Patterns (LBP) descriptor [6] is a histogram of quantized LBPs, which are extracted in a local image neighborhood.

We extract LBP descriptor and represent each fundus image using LBP bagof-words (BOW). Bag-of-words is to represent the image by using the frequency of the words. In this work, the training images are used to construct codebooks (words). We compute the LBP descriptor of each codebook image, and cluster them using K-means clustering method [9] to get the codebook centres. The frequency of the words is called LBP BOW. Here K is set to be 128 .

Histograms of Oriented Gradients The HOG features [5] are widely used in object detection study. Given an input image, the HOG features can be extracted using the following process:

- Decompose the given image into small squared patches.

- Compute an histogram of oriented gradients in each patch.

- Normalize the histogram using a block-wise pattern.

- Generate the final descriptor. 


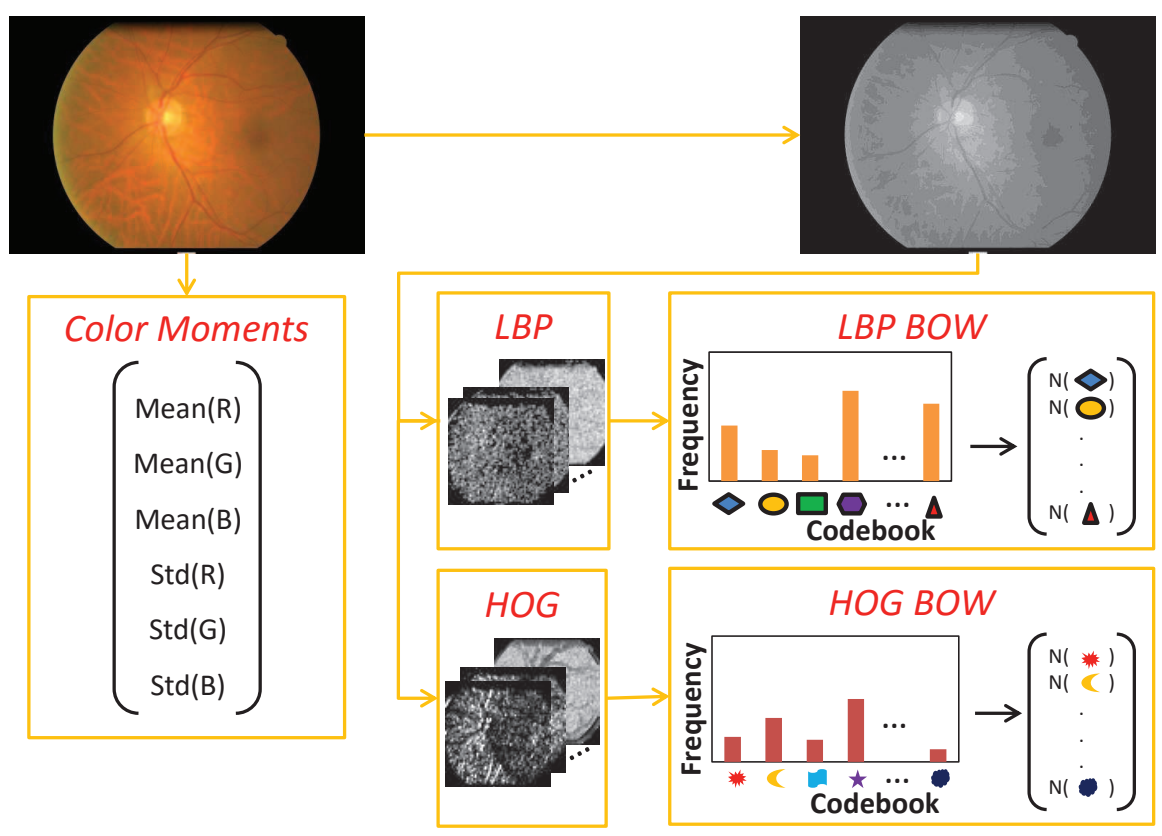

Fig. 3. Feature extraction scheme.

We extract HOG feature and quantized it using HOG bag-of-words. The same as LBP bag-of-words feature, we compute HOG feature of each training image, and get the codebook centres by using K-means clustering method. Here $\mathrm{K}$ is set to be 128 .

\subsection{Detection}

To evaluate the tessellated fundus detection system, we employ Support Vector Machine (SVM) [10] to classify the images into two groups, they are images with tessellated fundus and without tessellated fundus. Both linear and radial basis function(RBF) kernels are investigated. LIBSVM package [11] is used for classification in this experiment. For RBF kernel, 2-fold cross validation is applied to determine the proper SVM parameters.

\section{EXPERIMENTS}

\subsection{Dataset Construction}

A total of 836 fundus images collected from local hospital are used to construct the dataset. Among which, 418 images are positive samples, which are images with tessellated fundus; the other 418 images are negative samples, which are 


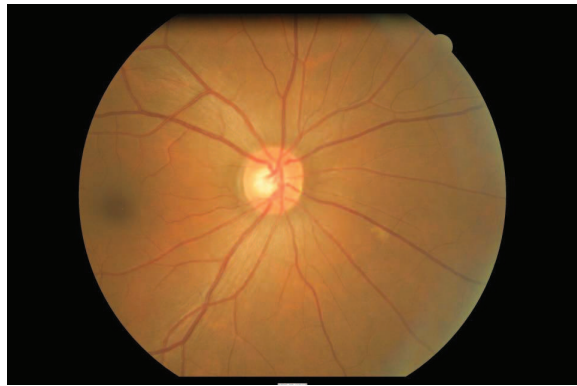

(a)

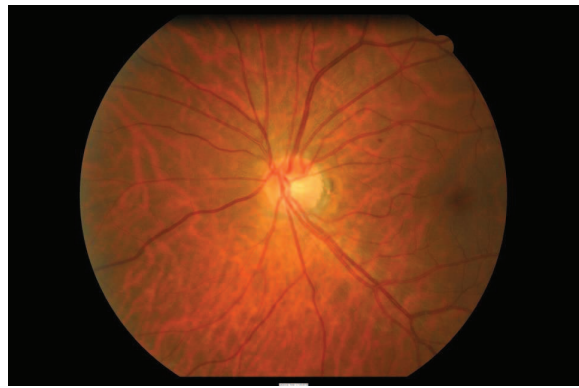

(c)

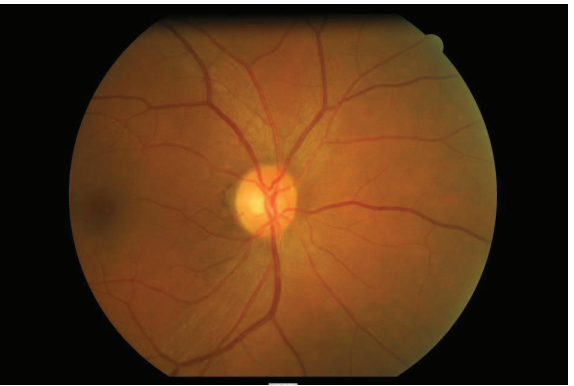

(b)

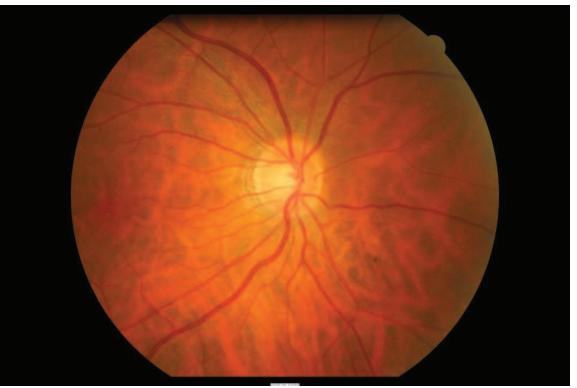

(d)

Fig. 4. Exemplar photographs in our dataset. (a) and (b) are negative samples (photographs without tessellated fundus), (c) and (d) are positive samples (photographs with tessellated fundus).

images without tessellated fundus. Fig. 4 shows exemplar images in our dataset. Here, (a) and (b) are negative samples (images without tessellated fundus), (c) and (d) are positive samples (images with tessellated fundus). As can be seen from Fig. 4, the choroidal vessels could be visually identified.

\subsection{Detection Results}

To evaluate the proposed method, we randomly divide the dataset into two subsets with equal size. One of the subset is used as training set, the other subset is used as testing set. Each subset includes 209 positive samples and 209 negative samples. As mentioned in the previous part, all codebooks are generated using training samples.

We report the performance on individual features and the combination of these features to justify the effectiveness of the individual features. Then sensitivity $P_{+}$, specificity $P_{-}$, and their average $P=\left(P_{+}+P_{-}\right) / 2$ are computed to evaluate the performance. Here, 


$$
\begin{gathered}
P_{+}=\frac{\# \text { True Positive }}{\# \text { Total Positive }} \\
P_{-}=\frac{\# \text { True Negative }}{\# \text { Total Negative }}
\end{gathered}
$$

Where \#True Positive denotes the number of correctly classified positive samples, \#Total Positive denotes the total number of positive samples in the testing set, \#True Negative denotes the number of correctly classified negative samples, and \#Total Negative denotes the total number of negative samples in the testing set. Table 1 shows the detection results.

Table 1. Detection Results.

\begin{tabular}{c|c|c|c|c|c}
\hline & & Color Moments & $L B P$ & $H O G$ & Color Moments + LBP $+H O G$ \\
\hline \hline Linear & Sensitivity $P_{+}$ & 0.52 & 0.99 & 0.96 & 0.99 \\
& Specificity $P_{-}$ & 0.78 & 0.97 & 0.90 & 0.98 \\
& Accuracy $P$ & 0.65 & 0.98 & 0.93 & 0.98 \\
\hline \multirow{2}{*}{ RBF } & Sensitivity $P_{+}$ & 0.50 & 0.99 & 0.95 & 0.99 \\
& Specificity $P_{-}$ & 0.74 & 0.92 & 0.81 & 0.95 \\
& Accuracy $P$ & 0.62 & 0.96 & 0.88 & 0.97 \\
\hline
\end{tabular}

As shown in Table 1, there is no significant difference between linear SVM and RBF SVM results for either single feature or feature combination. For single feature, texture features outperform color features, indicating that texture difference is more significant. By considering both texture features and color features, the feature combination gives the best performance.

Fig. 5 shows some correctly classified and wrongly classified images. The first row and second row shows correctly classified negative samples (without tessellated fundus) and correctly classified positive samples (with tessellated fundus), respectively. The failure cases are shown on the third row. From left to right, the first two images are negative samples which are wrongly classified as positive samples; the last two images are positive samples which are wrongly classified as negative samples. It can be seen that, all the failure cases are not extreme case, which are slightly similar to the opposite class.

\subsection{Codebook Size}

Fig. 6 shows the detection results for different codebook sizes. For both HOG and LBP features, as codebook size increases from 8 to 128, the detection accuracy increases to around $96 \%$. The performance shows no significant improvement as the codebook size increases further. By considering both efficiency and effectiveness, we set codebook size as 128 for both LBP and HOG features. 


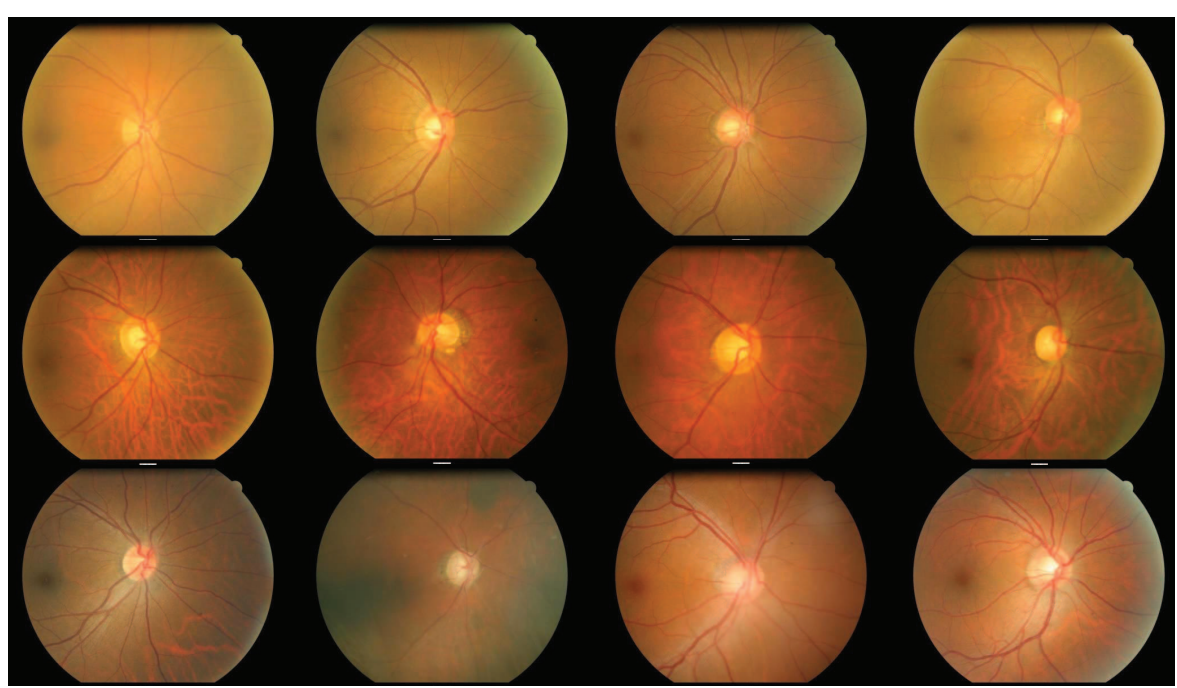

Fig. 5. Experimental results. The first row shows correctly classified negative samples (without tessellated fundus). The second row shows correctly classified positive samples (with tessellated fundus). The third row shows failure samples. For the failure cases, from left to right, the first two images are negative samples which are wrongly classified as positive samples; the last two images are positive samples which are wrongly classified as negative samples.

\section{DISCUSSIONS AND CONCLUSIONS}

In this work, we proposed an automated system for tessellated fundus detection. Color Moments, LBP and HOG features are studied and compared for the detection scheme. The detection results for SVM with linear and RBF kernels were also compared. The proposed method is able to achieve an average accuracy of $98 \%$ for linear SVM and $97 \%$ for SVM with RBF kernel, indicating that our proposed method is effective and reliable. To the best of our knowledge, this is the first automated tessellated fundus detection study. This work could be used as a preprocess for automated classification and grading for myopic maculopathy.

\section{References}

1. Adatia, F.A., Luong, M., Munro, M., Tufail, A.: The other cnvm: A review of myopic choroidal neovascularization treatment in the age of anti-vascular endothelial growth factor agents. survey of ophthalmology 60 (2015) 204-215

2. Ohno-Matsui, K., Kawasaki, R., Jonas, J.B., Cheung, C.M.G., Saw, S.M., Verhoeven, V.J., Klaver, C.C., Moriyama, M., Shinohara, K., Kawasaki, Y., et al.: International photographic classification and grading system for myopic maculopathy. American journal of ophthalmology 159 (2015) 877-883 

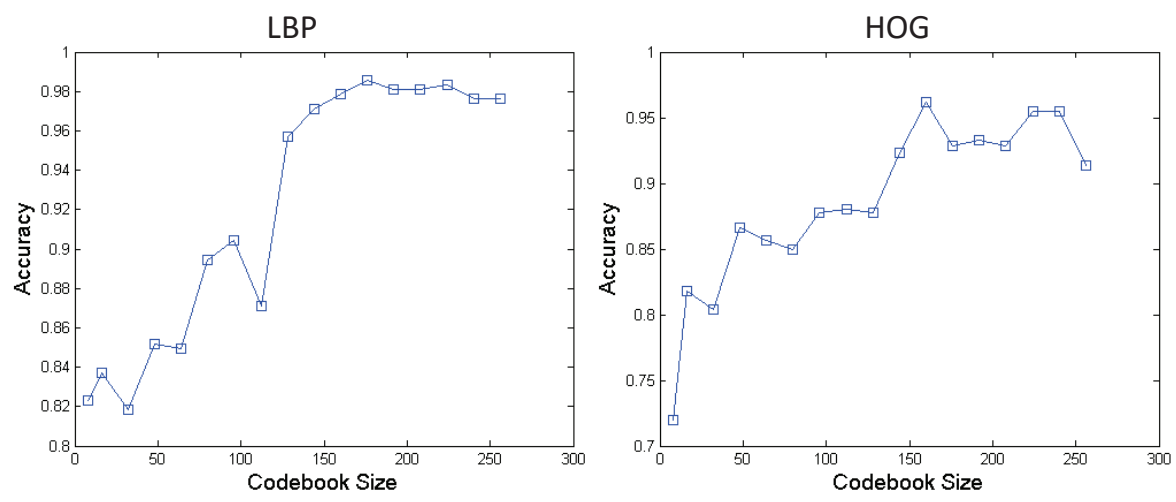

Fig. 6. Detection results for different codebook sizes.

3. Tan, N., Liu, J., Wong, D., Lim, J., Zhang, Z., Lu, S., Li, H., Saw, S., Tong, L., Wong, T.: Automatic detection of pathological myopia using variational level set. In: 2009 Annual International Conference of the IEEE Engineering in Medicine and Biology Society. (2009) 3609-3612

4. Cheng, J., Tao, D., Liu, J., Wong, D.W.K., Tan, N.M., Wong, T.Y., Saw, S.M.: Peripapillary atrophy detection by sparse biologically inspired feature manifold. IEEE transactions on medical imaging 31 (2012) 2355-2365

5. Dalal, N., Triggs, B.: Histograms of oriented gradients for human detection. In: Computer Vision and Pattern Recognition. Volume 1., IEEE (2005) 886-893

6. Muja, M., Lowe, D.G.: Fast approximate nearest neighbors with automatic algorithm configuration. VISAPP (1) 2 (2009) 2

7. Ojala, T., Pietikainen, M., Maenpaa, T.: Multiresolution gray-scale and rotation invariant texture classification with local binary patterns. IEEE Transactions on Pattern Analysis and Machine Intelligence 24 (2002) 971-987

8. Fei-Fei, L., Perona, P.: A bayesian hierarchical model for learning natural scene categories. In: Computer Vision and Pattern Recognition. Volume 2., IEEE (2005) $524-531$

9. Bishop, C.M.: Neural networks for pattern recognition. Oxford university press (1995)

10. Cortes, C., Vapnik, V.: Support-vector networks. Machine learning 20 (1995) 273-297

11. Chang, C.C., Lin, C.J.: LIBSVM: A library for support vector machines. ACM Transactions on Intelligent Systems and Technology 2 (2011) 27:1-27:27 Software available at http://www.csie.ntu.edu.tw/ cjlin/libsvm. 